\title{
Evolving
}

Technology

\section{Surgical aortic valve replacement after percutaneous aortic valve implantation: What have we learned?}

Pierre-Yves Litzler, MD, ${ }^{a}$ Alain Cribier, MD, ${ }^{\mathrm{b}}$ Alan Zajarias, MD, ${ }^{\mathrm{b}}$ Diane Comte, MD, ${ }^{a}$ Hélène Eltchaninoff, MD, Christophe Tron, MD, ${ }^{b}$ Catherine Haas-Hubscher, MD, ${ }^{c}$ and Jean-Paul Bessou, MD ${ }^{a}$

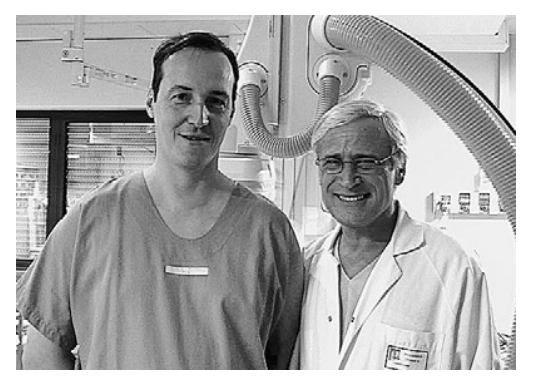

Drs Litzler and Cribier
Objective: We report the first case description of surgical aortic valve replacement after percutaneous valve implantation.

Methods: An 87-year-old man with severe aortic stenosis who was rejected for surgical intervention underwent percutaneous valve implantation through a retrograde femoral approach. The procedure was complicated by cardiogenic shock caused by severe aortic insufficiency, leading to emergency surgical aortic valve replacement.

Results: The operative findings revealed the presence of commissural paravalvular leaks and centrally malapposed leaflets. Surgical replacement was uneventful, and the patient was discharged on day 30 , despite a challenging postoperative course. His follow-up at 1 year has been uneventful. This case illustrates that overdilatation of the stent is not recommended because it might worsen central aortic insufficiency. Moreover, the transapical route should be considered when the appropriately sized prosthesis is unable to be inserted because of inappropriate vascular access. However, despite an initial "prohibitive" surgical risk, surgical aortic valvular replacement after percutaneous valve implantation could be easily performed.

Conclusion: Percutaneous heart valve implantation, which provides a larger surface area than balloon valvotomy, can be offered to patients with cardiogenic shock and severe comorbidities to improve their hemodynamic state and reduce their surgical

risk.
From the Departments of Thoracic and Car diovascular Surgery, ${ }^{\mathrm{a}}$ Cardiology, ${ }^{\mathrm{b}}$ and Anaesthesiology, ${ }^{\mathrm{c}}$ Rouen University Hospital Charles Nicolle, Rouen, France.

Supported by ARCTCV (Research Association-Cardiac Surgery Department-Rouen University Hospital-Rouen-France).

Alain Cribier reports consulting and lecture fees from Edward Lifesciences.

Received for publication Dec 5, 2007; accepted for publication Dec 24, 2007.

Address for reprints: Pierre-Yves Litzler, MD, Department of Thoracic and Cardiovascular Surgery, Rouen University Hospital, 1, rue de Germont, 76000 Rouen, France (E-mail: pierre-yves.litzler@ chu-rouen.fr).

J Thorac Cardiovasc Surg 2008;136:697701

\section{$0022-5223 / \$ 34.00$}

Copyright (C) 2008 by The American Association for Thoracic Surgery

doi:10.1016/j.jtcvs.2007.12.070
$\mathrm{P}$ ercutaneous strategies for the treatment of severe aortic stenosis in nonsurgical patients evolved over 20 years from balloon valvuloplasty to percutaneous heart valve (PHV) implantation. ${ }^{1}$ Despite encouraging results, this technique is still evolving, and each case provides a source of procedural improvement. We report the first case description of a surgical aortic valve replacement after percutaneous aortic valve implantation and discuss the findings revealed by this intervention.

\section{Clinical Summary}

An 87-year-old man with severe symptomatic aortic stenosis and in New York Heart Association functional class 3 or 4 was transferred to our institution in October 2006 for aortic PHV implantation. He was deemed a nonsurgical candidate by 2 independent surgeons because of his age and multiple comorbidities, which included previous stroke, ischemic cardiomyopathy with a left ventricular ejection fraction (LVEF) of $24 \%$, proximal occlusion of the left circumflex artery, atrial fibrillation with 


\section{Abbreviations and Acronyms}

$\mathrm{LVEF}=$ left ventricular ejection fraction

$\mathrm{PHC}=$ percutaneous heart valve

pacemaker dependency, and a prior right hemicolectomy for colon cancer. His logistic EuroSCORE was $22.78 \%$. A successful balloon aortic valvuloplasty had been performed 8 months prior, but his symptoms had recently recurred.

On admission, transthoracic echocardiographic analysis revealed a severely calcified aortic valve with a valve area of $0.4 \mathrm{~cm}^{2}$, a mean aortic pressure gradient of $46 \mathrm{~mm} \mathrm{Hg}$, an aortic annulus diameter of $20 \mathrm{~mm}$, a pulmonary artery systolic pressure of $60 \mathrm{~mm} \mathrm{Hg}$, and an LVEF of $25 \%$. Aortic angiographic analysis with iliofemoral runoff and computed tomographic scanning revealed severe calcification of the distal aorta and proximal iliac arteries. The diameter of the iliofemoral vessels did not exceed $8 \mathrm{~mm}$. The patient was included in the Registry of EndoVascular Implantation of Valves in Europe (REVIVE) trial and was scheduled to receive a 23-mm Cribier-Edwards PHV (Percutaneous Valve Technologies; Edwards LifeScience, Irvine, Calif) by using a 22F delivery system through the right femoral artery.

The incision of the groin was performed after achievement of local anesthesia, and the right femoral artery was catheterized. Uneventful aortic valve predilatation with a $23-\mathrm{mm}$ Z-Med II (Numed, Cornwall, Canada) balloon through a $14 \mathrm{~F}$ sheath was performed. After progressive dilatation of the right femoroiliac arteries with $16 \mathrm{~F}$ to $22 \mathrm{~F}$ dilators, the 23-mm Cribier-Edwards prosthesis was advanced to the aortic valve and placed under rapid cardiac stimulation (200 beats $/ \mathrm{min}$ ). After this procedure, recovery of a satisfactory blood pressure was only obtained with the use of inotropes and vasopressors. Supra-aortic angiographic analysis subsequently showed severe aortic regurgitation (Figure 1). Postdilatation of the PHV with a 23-mm balloon was performed, leading to a period of cardiac arrest requiring several minutes of chest compressions. The patient was intubated, mechanically ventilated, and transferred to the intensive care unit with escalating vasopressor requirements. Although he was extubated 6 hours later, he persisted with hemodynamic instability characterized by a diastolic blood pressure of $35 \mathrm{~mm} \mathrm{Hg}$ and oliguria. Repeat echocardiographic analysis confirmed the presence of severe paravalvular insufficiency with 2 distinct jets and a smaller area of mild central aortic insufficiency. The aortic valve area was measured at $1.7 \mathrm{~cm}^{2}$ with a minimal transvalvular gradient and an LVEF of $60 \%$ with inotropes.

Because of his poor prognosis and frank improvement of cardiac function, after obtaining consent from the patient and his family, surgical aortic valve replacement was attempted.

After a median sternotomy, the patient was started on cardiopulmonary bypass. Anterograde and retrograde continuous hot blood cardioplegia was used for myocardial

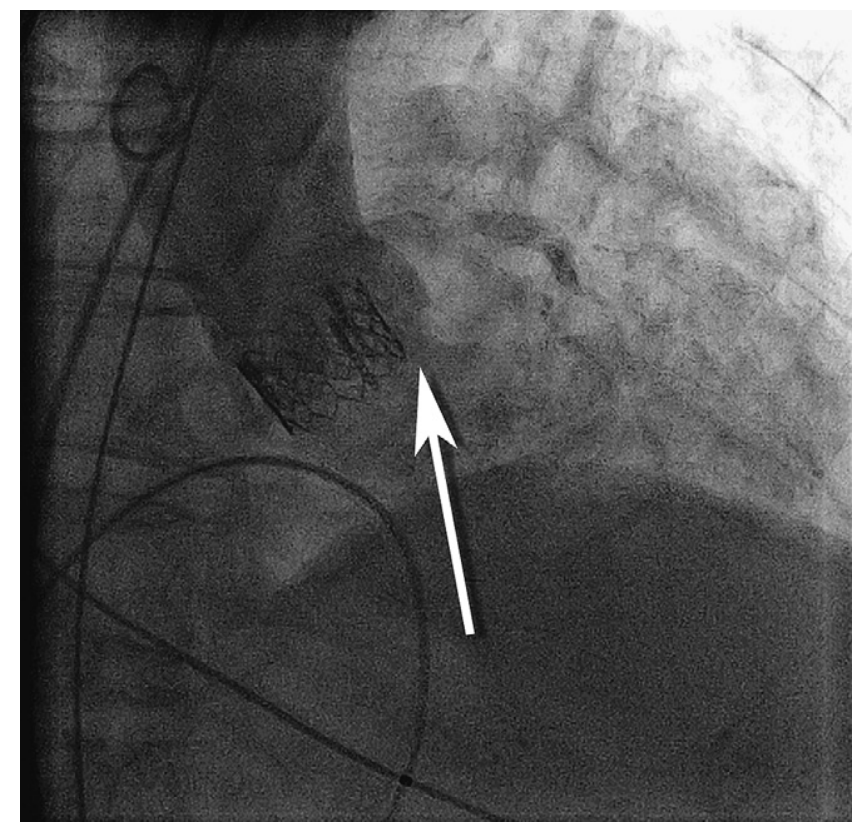

Figure 1. Supra-aortic angiogram showing commissural paravalvular leak (arrow).

protection. Incision of the ascending aorta revealed a PHV in perfect position on the aortic annulus with the coronary ostia free from occlusion (see video, online). Analysis of the native valve showed a major gap between the stent and the commissure between the left and right coronary cusps and a minor one between the stent and the commissure between the left and noncoronary cusps (Figure 2). There was a small central leak on the PHV caused by leaflet malapposition, leading to central dehiscence. Nevertheless, the circular

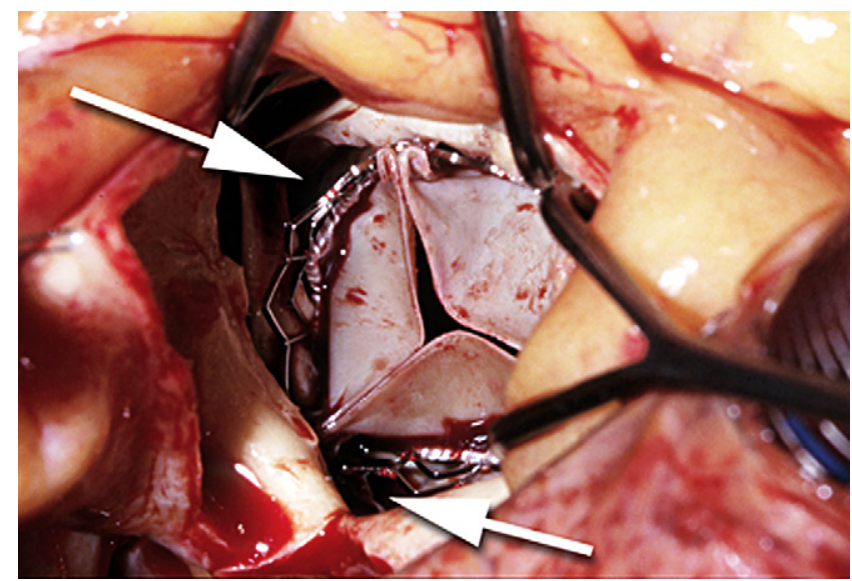

Figure 2. Operative view demonstrating the presence of paravalvular leaks on the commissures between the left and right coronary cusps and between the left and noncoronary cusps (arrows). The valve presents a geometric asymmetry of the 3 cusps, leading to a dehisced prosthesis with a small central incompetence. 


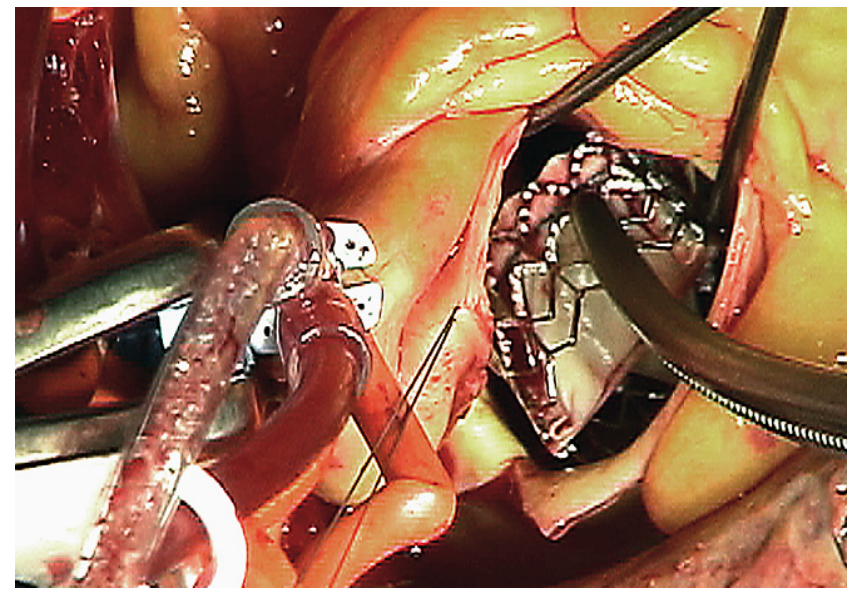

Figure 3. Percutaneous heart valve removal with a Kelly forceps.

shape of the prosthesis was well respected. Removal of the PHV was performed without difficulty by pulling the prosthesis with a Kelly forceps (Figure 3). The 3 native aortic valve leaflets remained retracted in an open position because of commissural and calcium cracking after repetitive balloon inflation and stent deployment (Figure 4).

Resection of the cusps was problematic. The valve was heavily calcified, and the successive balloon valvuloplasties had disrupted numerous calcified nodules. The calcareous fragments were carefully removed from the cusps, aortic annulus, and mitral valve leaflets. Because of the risk of embolization, the implantation site and left ventricular cavity were extensively washed. The size of the aortic annulus was tested, and a 23-mm Carpentier-Edwards Perimount valve (Edwards LifeScience) was implanted uneventfully. After 85 minutes, the cardiopulmonary bypass was weaned while the patient required $18 \mu \mathrm{g} \cdot \min ^{-1} \cdot \mathrm{m}^{-2}$ dobutamine and $0.7 \mu \mathrm{g} \cdot \mathrm{min}^{-1} \cdot \mathrm{m}^{-2}$ norepinephrine. Hemostasis was difficult because of the antiplatelet treatment previously administered (aspirin and clopidogrel).

The postoperative course was marked by 3 days of anuria requiring dialysis after cardiogenic shock and contrastinduced nephropathy. The patient was extubated, progressively weaned off of inotropic support, and transferred out of the intensive care unit after 8 days. Postoperative echocardiographic analysis revealed no aortic insufficiency, a mean aortic gradient of $11 \mathrm{~mm} \mathrm{Hg}$, pulmonary artery systolic pressure of $30 \mathrm{~mm} \mathrm{Hg}$, and an LVEF of 54\%. The patient was discharged from the hospital on day 30 . He is still alive 1 year after the procedure with improved physical activity and without recurrence of heart failure.

\section{Discussion}

Surgical aortic valve replacement has a very low mortality and morbidity and is considered the gold standard for the treatment of symptomatic aortic valve stenosis. ${ }^{2}$ Neverthe-

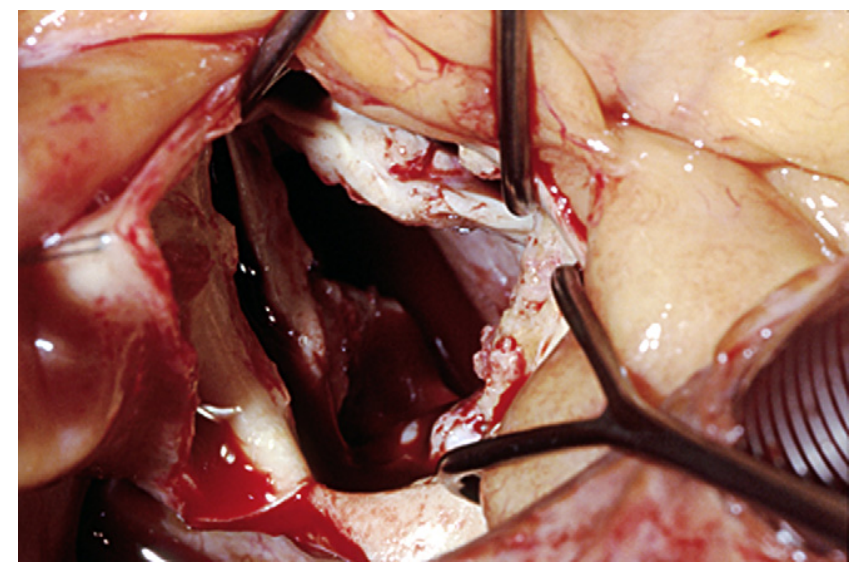

Figure 4. Operative view after removal of the percutaneous heart valve showing the calcified cusps, which remained open because of the rupture of the valve calcifications.

less, a nonnegligible cohort of patients is not referred to or is rejected for surgical intervention because of their comorbidities. Most of these patients are considered to have an "unacceptable" high risk for cardiac surgery, as calculated by using the Society of Thoracic Surgeons risk calculator or logistic EuroSCORE. The development of PHV implantation appears to be a solution to treat these high-risk patients. However, percutaneous valvular replacement still requires large arterial sheaths that limit patient candidacy.

Aortic PHV sizing is guided by echocardiographic measurement of the aortic annulus. Although this patient's aortic annulus diameter $(20 \mathrm{~mm})$ was compatible with a $23-\mathrm{mm}$ valve, early experience with PHV implantation has revealed a higher rate of periprosthetic regurgitation with the use of the $23-\mathrm{mm}$ valve when compared with the $26-\mathrm{mm}$ valve, particularly in male patients. The $24 \mathrm{~F}$ sheath, required for the 26-mm valve, has a 9-mm outer diameter. Its insertion would have increased the risk of vascular damage caused by the arterial calcification and the iliac artery's minimal luminal diameter $(8 \mathrm{~mm})$. PHV placement after achievement of local anesthesia, as performed in our institution, appears advantageous because it alerts the implanting physician of patient discomfort during arterial dilation or sheath insertion, which suggests arterial damage. Such an occurrence forces the operating team to stop the procedure, re-evaluate the possibility of placing a smaller valve, or seek an alternative placement route. This has minimized the incidence of arterial dissection or rupture.

During surgical intervention, we observed that the paravalvular leaks found on the angiograms were predominantly located on 2 of the 3 commissures of the native valve. Severe valvular calcification limits the full circular expansion of the delivery balloon catheter, adding to the degree of aortic insufficiency. Despite the rupture of numerous calcifications and full balloon expansion, complete circumferential apposition 
between the aortic annulus and the stent prosthesis is not always achieved. This phenomenon can lead to paravalvular leaks and early prosthetic degeneration. The presence of major paravalvular leaks has a noxious hemodynamic effect in these fragile patients. The hypertrophied heart with diastolic dysfunction is unable to cope with the resultant severe acute aortic insufficiency, which leads to immediate hemodynamic failure. Minor paravalvular insufficiencies, as are generally observed in these procedures, are well tolerated because of the improvement of myocardial contraction after significant afterload reduction.

As seen after the aortotomy (Figure 2), the valve presented geometric asymmetry in the 3 cusps leading to a central malapposition of the valve leaflets. Central dehiscence is a wellknown mode of failure of surgically implanted bioprostheses and is the only one that is age independent. ${ }^{3}$ This phenomenon is generally related to an irregular distribution of the sutures. In our case this could be directly linked to overdilation after the procedure of implantation or to an asymmetric stent deployment caused by the presence of heavy calcifications during the procedure.

The first-generation bovine stented pericardial valves were marked by early failure related to the basal attachment site of the cusps and the supporting stent. This was particularly observed in the "alignment stitch" zone, which maintains proper position of the valve leaflets and their apposition at each commissure. ${ }^{4}$ All improvement of the prosthesis design to diminish paravalvular leaks or improve the leaflet apposition could hopefully lead to an increase of its durability. The discrepancy observed between the aortic annulus and the size of the PHV delivery system required in the patient supports the use of a transapical approach in certain cases to avoid a mismatched prosthesis. Unfortunately, this technique was unavailable at the time of implantation.

It is important to note that despite an initial "prohibitive" surgical risk, surgical aortic valve replacement after percutaneous valve implantation was performed. The removal of the PHV was simple, despite the impaction of the stent in the cusps of the native valve. The radial force of the stent is easily overcome, and nonexcessive tension is necessary to extract the valve prosthesis within seconds. The disruption of the calcifications might give rise to the only difficulty of this operation. Indeed, the resection of the 3 cusps produces innumerable numbers of fragments, which increase the risk of embolization after aortic cross-clamp removal. Copious irrigation is necessary to obtain a secure clean field before the implantation of the new bioprosthesis. The low rate of stroke observed during implantation of the $\mathrm{PHV}^{1}$ is probably due to the capture of the disrupted calcifications by the stent.

In our patient a $25-\mathrm{mm}$ tester valve seemed oversized, and the $23-\mathrm{mm}$ prosthesis fit perfectly in the native annulus after extensive decalcification. Thus we replaced the 23-mm PHV with a 23-mm Edwards Perimount valve. PHVs are sized by means of an echocardiographic measurement at the cusp insertion point in the aortic annulus, and they are mounted on a thin stent platform. The measurement obtained with the valve tester reflects the diameter of the prosthesis and its casing, representing a different dimension. We must underline that despite different designs, these valves present a similar effective orifice area of $1.7 \mathrm{~cm}^{2}{ }^{1,5}$ The resection of the cusps and the annulus decalcification allowed the use of a largerprofile prosthesis.

Despite severe comorbidities, hemodynamic compromise, and a logistic EuroSCORE of $22.78 \%$, the postoperative course had complications that were easily managed. In patients with multiple comorbidities, the logistic Euroscore is inaccurate and overestimates their surgical mortality rate, thus limiting its usefulness. ${ }^{6}$ Clinical judgment is crucial for rendering a surgical opinion and should take precedence to risk models in this patient population. The use of risk calculators should be used in clinical trials to unify a patient population. ${ }^{7}$ In clinical practice their use complements clinical judgment.

A PHV might be used as a bridge for cardiac surgery, replacing balloon valvotomy, as suggested in the American College of Cardiology/American Heart Association "2006 Guidelines for the management of patients with valvular heart disease." "2 As noted earlier, PHV implantation provides a larger surface area than balloon valvuloplasty, which rarely exceeds $1 \mathrm{~cm}^{2}$. PHV implantation can be offered to patients with refractory pulmonary edema or cardiogenic shock to improve their hemodynamic state and reduce their surgical risk. Other patients with severe comorbidities that might limit their survival could also benefit from this new device. The viability of such algorithms remains to be demonstrated.

\section{Conclusion}

PHV implantation is at an early stage of development. The encouraging results obtained with preliminary implantations should not underestimate the complexities and tribulations that arise during the development of new techniques. The future of percutaneous procedures depends on the full collaboration between the cardiac surgeon, cardiologist, and anesthesiologist to manage these challenging patients.

\section{References}

1. Cribier A, Eltchaninoff H, Tron C, Bauer F, Agatiello C, Nercolini D, et al. Treatment of calcific aortic stenosis with the percutaneous heart valve: mid-term follow-up from the initial feasibility studies: the French experience. J Am Coll Cardiol. 2006;47:1214-23.

2. Bonow RO, Carabello BA, Kanu C, de Leon AC Jr, Faxon DP, Freed MD, et al. ACC/AHA 2006 guidelines for the management of patients with valvular heart disease: a report of the American College of Cardiology/ American Heart Association Task Force on Practice Guidelines (writing committee to revise the 1998 Guidelines for the Management of Patients With Valvular Heart Disease). Developed in collaboration with the Society of Cardiovascular Anesthesiologists. Endorsed by the Society for Cardiovascular Angiography and Interventions and the Society of Thoracic Surgeons. Circulation. 2006;114:e84-231. 
3. Roselli EE, Smedira NG, Blackstone EH. Failure modes of the Carpentier-Edwards pericardial bioprosthesis in the aortic position. J Heart Valve Dis. 2006;15:421-8.

4. Schoen FJ, Fernandez J, Gonzalez-Lavin L, Cernaianu A. Causes of failure and pathologic findings in surgically removed Ionescu-Shiley standard bovine pericardial heart valve bioprostheses: emphasis on progressive structural deterioration. Circulation. 1987;76:618-27.

5. Eichinger WB, Botzenhardt F, Keithahn A, Guenzinger R, Bleiziffer S, Wagner I, et al. Exercise hemodynamics of bovine versus porcine bioprostheses: a prospective randomized comparison of the Mosaic and Perimount aortic valves. J Thorac Cardiovasc Surg. 2005;129:1056-63.
6. Shanmugam G, West M, Berg G. Additive and logistic EuroSCORE performance in high risk patients. Interact Cardiovasc Thorac Surg. 2005;4: 299-303.

7. Vassiliades TA Jr, Block PC, Cohn LH, Adams DH, Borer JS, Feldman T, et al. The clinical development of percutaneous heart valve technology: a position statement of the Society of Thoracic Surgeons (STS), the American Association for Thoracic Surgery (AATS), and the Society for Cardiovascular Angiography and Interventions (SCAI) endorsed by the American College of Cardiology Foundation (ACCF) and the American Heart Association (AHA). J Am Coll Cardiol. 2005; 45:1554-60. 